\title{
PREDICTIVE CONTROL OF THE IRON ORE BENEFICIATION PROCESS BASED ON THE HAMMERSTEIN HYBRID MODEL
}

\author{
Olga PORKUIAN, Vladimir MORKUN, ," Natalia MORKUN," Oleksandra SERDYUK"*
}

\author{
${ }^{*}$ Faculty of Information Technology and Electronics, Department of Programming and Mathematics, \\ Volodymyr Dahl East Ukrainian National University, pr. Central 59-a, Severodonetsk, 93400, Ukraine \\ **Faculty of Information Technologies, Computer Science and Technology Department, Kryvyi Rih National University,
} 11 Vitaliy Matusevych St., Kryvyi Rih, 50027, Ukraine

oporkuian@gmail.com, morkunv@gmail.com, nmorkun@gmail.com, o.serdiuk@i.ua

received 2 August 2019, revised 13 January 2020, accepted 15 January 2020

\begin{abstract}
Non-linear, dynamic, non-stationary properties characterize objects of the iron ore beneficiation line. Therefore, for their approximation, it is advisable to use models of the Hammerstein class. As a result of comparing the three models of Hammerstein: simple, parallel and recursive-parallel, it was shown that the best result for identifying the considered processes of magnetic beneficiation of iron ore by the minimum error criterion was obtained using the Hammerstein recursive-parallel model. Hence, it is recommended for the identification of beneficiation production objects.
\end{abstract}

Key words: Predictive control, iron ore, beneficiation, Hammerstein model, recursive-parallel model

\section{INTRODUCTION}

Providing metallurgical production with high-quality raw materials remains an urgent problem. The growth in mineral consumption requires the expansion of the raw material base, ensuring the necessary quality of raw and marketable ore, and increasing the profitability of mining and processing enterprises (Abonyi et al., 2000; Babuska, 1998). Over the past 20-30 years, the iron content in the extracted ore at the mining enterprises of Ukraine decreased by an average of $12.9 \%$ (Babuska, 1998; Babuska, 1998). Considering the current characteristics of raw materials processed at processing plants and the state of technological equipment when forming control actions in automatic control systems allows optimizing the technological process and increasing its technical and economic indicators. Thus, increasing the iron content in the concentrate from 64 to $65 \%$ allows in the sintering redistribution - to reduce ore consumption by $29-32 \mathrm{~kg} / \mathrm{t}$ and fluxes - by $30 \mathrm{~kg} / \mathrm{t}$; in the metallurgical division - to reduce the consumption of coke by $2.6-2.8 \%$, to increase the relative productivity of the blast furnace by $4.5-5 \%$. All this helps to reduce the total cost of iron production by about 4\% (Abonyi et al., 2000). This work aims to increase the efficiency of the automated control of the magnetic beneficiation processes of iron ores by developing theoretical bases and building predictive control of these processes based on Hammerstein models. Based on the results of the study and analysis of the problem of increasing the efficiency of automated control of iron ore beneficiation processes, the control concept and research objectives are formulated:

- to develop and study the models (in the class of Hammerstein models) and methods for the rapid identification of iron ore beneficiation processes as nonlinear dynamic objects, considering the interchangeability of their properties;
- to develop algorithms for predictive control of iron ore beneficiation processes based on the Hammerstein models.

The object of the study is dynamic technological processes and transformations in the presence of fuzzy and incomplete information that occurs during the concentration of iron ores, methods, and systems for automatic control of these processes. The subject of the research is models and algorithms for identifying nonlinear dynamic objects, predictive control algorithms, an automated process control system (APCS) of iron ore beneficiation. An analytical review of the work on the problems of automatic control of iron ore beneficiation processes showed that under conditions when the ore characteristics and the condition of technological equipment change, the final performance of the processing plant is mostly dependent on the efficiency of automatic control of technological units, which is primarily determined by its quality information and algorithmic support. Effective control requires the constant and rapid provision of a significant amount of data on the state of the control object (the content of the useful component in the product, the particle size distribution of the pulp at various points in the process, the concentration of the solid phase and pulp density, etc.), as well as high-speed algorithms for their processing with the aim of identification of the object and definition of control actions.

The same tendency also occurs in mining enterprises of other countries. Reducing the metal content in raw ore increases the resource intensity of production and the cost of finished products. To solve the problems of the synthesis of automatic control systems of nonlinear non-stationary objects, operating under severe restrictions, which are imposed on state and control variables, the Model Predictive Control method (MPC) (Zubov, 2006) and its modifications - Adaptive Model Predictive Control have recently been actively used (Sanches and Rodellar, 1996). Simplified, the idea of these methods is to use the variable values extrapolated to 
a certain depth in such a way as to minimize the future deviation of the system from the desired state during the formation of the control law, and thereby, ensure optimal control. At present, the Predictive Control paradigm has achieved such successes, including in industrial applications, which is considered by many authors as an alternative to PID regulation for complex multidimensional systems (Rossiter, 2003).

In (Zubov, 2006; Morkun and Tcvirkun, 2014; Sanches and Rodellar, 1996; Rossiter, 2003; Morkun et al., 2014a), various control schemes based on MPC are presented. The variant of the control structure proposed in Morkun et al. (2014a) allows multicriteria optimization with ranking criteria by priority. Most control facilities at the processing plants have both dynamic and nonlinear properties. The identification of such objects often causes significant difficulties. Some methods for the identification of nonlinear dynamic objects are considered in Ivanov (1991), Morkun et al. (2015a), Morkun et al. (2014b), Yucai (1999), Leontaritis and Billings (1987), Stoica (1981) and Morkun et al. (2018). As the analysis of these works shows, a common technique for identifying dynamic nonlinearity is the artificial separation of these two properties. The object is represented by a set of nonlinear static and linear dynamic blocks. In this case, N. Wiener proposed to consider a sequential connection in the following order: a dynamic block, followed by nonlinearity (Morkun et al., 2014c; Li and Shchetsen, 1968; Yucai,1999; Stoica, 1981). An alternative variant is a nonlinear block, followed by a dynamic one, proposed by Hammerstein and was considered in Shi and Sun (1990), Ivanov (1995), Narendra and Gallman (1966), Leontaritis and Billings (1987), Sjoberg (1995) and Stoica (1981).

In this case, a simple sequential Wiener model turns out to be more profitable for about half of the real objects than a simple Hammerstein model (Morkun et al., 2015b). However, in such a simple version, Wiener and Hammerstein models are used extremely rarely. In most cases, the object of identification is approximated by various combinations of these simple models. In this case, as shown by the studies carried out by the authors (Morkun et al., 2015b; Shi and Sun, 1990; Ivanov, 1995; Verhaegen and Westwick, 1996), parallel and recursive-parallel models turned out to be the best by the criterion of 'simplicity-quality'. These models are a parallel connection of single-type simple links. In papers (Morkun et al., 2015b; Shi and Sun, 1990), an essential feature of parallel models with an unlimited number of parallel branches was emphasized. The use of this class of models eliminates the solution of a complicated problem of choosing a model structure.

The question of choosing the parallel model structure is no longer determined by the accuracy of the model, but by the possibilities for effectively identifying its parameters. In this regard, the parallel Hammerstein model turns out to be more profitable, since it allows one-dimensional orthogonal identification algorithms (Ivanov, 1991), which do not impose any severe restrictions on the type of input test actions. The one-dimensional algorithms for identifying the parameters of the parallel Wiener model are not orthogonal (Young, 1977). Orthogonal algorithms for Wiener models are multidimensional and require significant computational resources.

In principle, it is possible to use not only parallel models but also sequential models containing a set of series-connected simple models of Hammerstein or Wiener. However, this approach is not used in practice since it has proven difficult to formalize and is associated with simulation modeling, which requires significant computational resources (Morkun et al., 2015b).

Recently, scientific papers have been published, which offer various options for increasing the efficiency and ease of use of Hammerstein models. So, in Ikhouane and Girib (2014) a unified framework for the identification of Wiener and Hammerstein systems that is valid for SISO and MIMO systems, discrete- and continuous-time settings, and with the only a priori knowledge that the system belongs to the set including Wiener and Hammerstein models is presented. The paper Ozer et al. (2016) studies the application of system identification of the Hammerstein model, which is a cascade of nonlinear second-order Volterra and a linear FIR model. The recursive least squares algorithm is used to determine the parameters of the proposed Hammerstein model. In (Rébillat et al., 2010), it is shown that the cascade of Hammerstein models makes it possible to describe a large class of nonlinearities conveniently. A simple method is proposed based on the phase property of exponential sinusoids for identifying the structural elements of such a model using only one measured system response. Ma et al. (2016) discusses the problem of estimating the state and parameters for a class of Hammerstein state space systems with a time delay. Both process noise and measurement noise are taken into account in the system. Based on the observed space form of canonical states and the separation of critical terms, a pseudo-linear regressive identification model is obtained. For unknown states in the information vector, the Kalman filter is used to search for optimal state estimates. Least squares algorithms based on the Kalman filter and recursive least squares algorithms are proposed. In Chen and Wang (2015), the problem of parameter identification for a Hammerstein system with continuous nonlinearity is studied. Taking into account the unknown structure of continuous nonlinearity, a Weierstrass approximation theorem is introduced to simplify nonlinearity. Then a stochastic gradient algorithm and an algorithm for optimizing a swarm of particles to estimate all unknown parameters of the Hammerstein system are proposed. In Chen and Ding (2015), the hierarchical least-squares algorithm is developed using the principle of hierarchical identification, which decomposes the nonlinear system into several subsystems with smaller sizes and fewer variables and estimates the parameters of each subsystem, respectively.

Importantly, the degree of adequacy of the model obtained is determined mainly by considering different types of uncertainty in the identification of the object. A significant error in the input data leads to an error in the calculation of the target function and, consequently, to a significant area of uncertainty in the choice of optimal control and optimal operation of the system. In Tobi and Hanafusa (1991), it was shown that only the representation of several constraints as fuzzy makes it possible to obtain a stable solution under conditions of inaccuracy of information and fuzziness of production constraints, with an indication of a reduced degree of admissibility of this mode, that is, as membership functions. Setting the problem in fuzzy form also significantly reduces the possibility of obtaining incompatible solutions for the calculation and optimization. In Abba et al. (2019), Hammerstein-wiener $(H W)$, general regression neural network (GRNN), and non-linear autoregressive with exogenous (NARX) neural network, leastsquare support vector machine (LSSVM) models were employed for multi-parametric (Hardness (mg/L), turbidity (Turb) $(\mu \mathrm{s} / \mathrm{cm}), \mathrm{pH}$ and suspended solid (SS) (mg/L)). The comparison of the results of modeling showed that HW served as the best model for the simulation of Hardness, Turb, and SS. Mete et al. (2016) present the Hammerstein model, which is obtained by cascade form of a nonlinear second-order Volterra (SOV) and a linear FIR model. Besides, the proposed Hammerstein model is optimized with a differential evolution algorithm (DEA). In Le et al. (2012), the 
recursive identification of Hammerstein structures is investigated. A recursive algorithm is then developed to address the limitations in the approaches currently available. The linear and nonlinear parameters are separated and estimated recursively in a parallel manner, with each updating algorithm using the most up-to-date estimation produced by the other algorithm at each time instant. Chen and Wang (2015) study the parameter identification problem for a Hammerstein system with continuous nonlinearity. Considering the unknown structure of the continuous nonlinearity, the Weierstrass approximation theorem is introduced to simplify the nonlinearity. Then a stochastic gradient algorithm and a particle swarm optimization algorithm are proposed to estimate all the unknown parameters of the Hammerstein system.

In Falck et al. (2012), consider the identification of WienerHammerstein systems using the Least-Squares Support Vector Machines based models. Their study illustrates that black-box models are a suitable technique for the identification of WienerHammerstein systems. Wills A. and Ninness (2012) examine the use of a so-called 'generalized Hammerstein-Wiener' model structure that is formed as the concatenation of an arbitrary number of Hammerstein systems. This approach is profiled using a Wiener-Hammerstein Benchmark example, which illustrates it to be effective and, via Monte-Carlo simulation, relatively robust against capture in local minima. Piroddi et al. (2012) analyse the performance of several black-box nonlinear model identification techniques for input-output models with polynomial nonlinearities on a benchmark identification problem.

The number of works devoted to the construction of fuzzy models, fuzzy control, and its various aspects is growing steadily, an example of some of which may be Tobi and Hanafusa (1991), Abonyi et al. (2000), and Postlethwaite (1996). The TakagiSugeno models have found wide application for the approximation of nonlinear systems, some examples of which were considered in Babuska (1998), Babuska (1998), Morkun et al. (2015c), and Kazuo and Wang (2001), in particular, there is a positive experience of using them to control individual objects of iron ore processing lines (Morkun and Tcvirkun, 2014; Morkun et al., 2015c).

\section{MATERIALS AND METHODS}

A significant role in the application of MPC approaches is performed by the model, based on which the future behaviour of the system is calculated. In order to make full use of the control capabilities using the MPC theory, it is necessary to provide an effective and fast identification of the characteristics of the control object, which will allow forming a model of this object quickly.

Therefore, when developing effective process control systems, the following essential aspects are relevant:

- development of new control algorithms and identification methods that make it possible to effectively use the information obtained, as well as considering the inevitable uncertainty and inaccuracy in the description of the control object;

- improvement of information support (improvement of measurement accuracy, the ability to measure additional parameters in the course of process control, the efficiency of information provided for control).

It should be noted that in recent years there has been a sharp increase in the number of studies devoted to the extraction of the fullest possible information about the control object (Ozer et al., 2016; Ma et al., 2016; Chen and Wang, 2015; Chen and Ding,
2015). To fulfil the goals of control, it is currently possible to use information not only in a formalized numerical form but also in linguistics, in the form of expert conclusions. Such work initiated intensive research based on the use of new mathematical applications in the field of identification and control.

The ore beneficiation processes are spatially distributed multidimensional automation objects with a complex, multi-connected structure. The beneficiation technological lines usually consist of several successively arranged stages, each of which includes the following main technological operations: grinding, classification, magnetic separation. The technological processes of ore processing plants provide for multistage crushing and grinding of ore to prepare it for the next separation (Morkun et al., 2014b; Morkun et al., 2015a; Morkun et al., 2018). The purpose of these operations is to reveal ore aggregates and to isolate fractions of various minerals from each other by reducing the size of mineral grains to $0.1 \mathrm{~mm}$ or less. As control objects, grinding units can be represented in the form of some operators that convert the vectors of input variables into vectors of output parameters. In turn, the output parameters of the grinding process must be considered as input for the next stage of the beneficiation process. Elements of the vectors of the output parameters of the grinding department are their qualitative and quantitative indicators. The main quantitative indicators are feed productivity and finished class size productivity. The quality of the crushed product is characterized by the density or solid content in the pulp, the granulometric composition of the solid phase, as well as the quality indicators of the initial ore (content of useful components, mineral composition, etc.), not depending on the technological mode of operation of the grinding cycle. Thus, the elements of the vector of output parameters formed by the grinding redistribution (productivity and particle size distribution of the product) can be taken as control actions in solving problems of optimizing the beneficiation process.

Let's consider the synthesis of control based on Hammerstein models for the first stage of iron ore beneficiation as an integral part of the entire iron ore beneficiation processing line, which makes a decisive contribution to the final result. As shown in Ozer et al. (2016), Morkun et al. (2014b), Sanches and Rodellar (1996), and Narendra and Gallman (1966), most technological devices of this stage of beneficiation correspond in their properties to the models of Hammerstein. The generalized control scheme is presented in Fig. 1.

The following notation was used: 1 - mill, 2 - classifier, 3 - magnetic separator, 4 - conveyor, 5 - bunker, 6 - drive motor, 7 - water flow valve, 8 - ore supply control system, 9 - the classifier water supply control system, 10 - an identification subsystem, 11 - a driver of control actions, 12 - an optimizer, 13 - a sensor of industrial product parameters, 14 - an ore consumption sensor; 15 - information support subsystem, 16 - pulp parameter sensor.

The Hammerstein model, on the basis of which the predictive control will be implemented, is a combination of a fuzzy nonlinear block and a crisp linear dynamic block. Thus, the term 'hybrid model' corresponds to the structure of the model.

Let a static nonlinear block represents the object of study with an input vector $\mathrm{u}=\left[\mathrm{u}_{1}, \ldots, \mathrm{u}_{\mathrm{n}}\right]^{\mathrm{T}}$, a vector of converted input variables $\mathrm{v}=\left[\mathrm{v}_{1}, \ldots, \mathrm{v}_{\mathrm{n}}\right]^{\mathrm{T}}$, related to $\mathrm{u}_{\mathrm{h}}$ according to

$v_{h}=f_{h}\left(u_{h}\right), h=1, \ldots, n$,

where, $f_{h}\left(u_{h}\right)$ are the functions characterizing nonlinear block by control $h$-channel, and a dynamic linear block with a vector of output variables $y=\left[y_{1}, \ldots, y_{m}\right]^{T}$. 


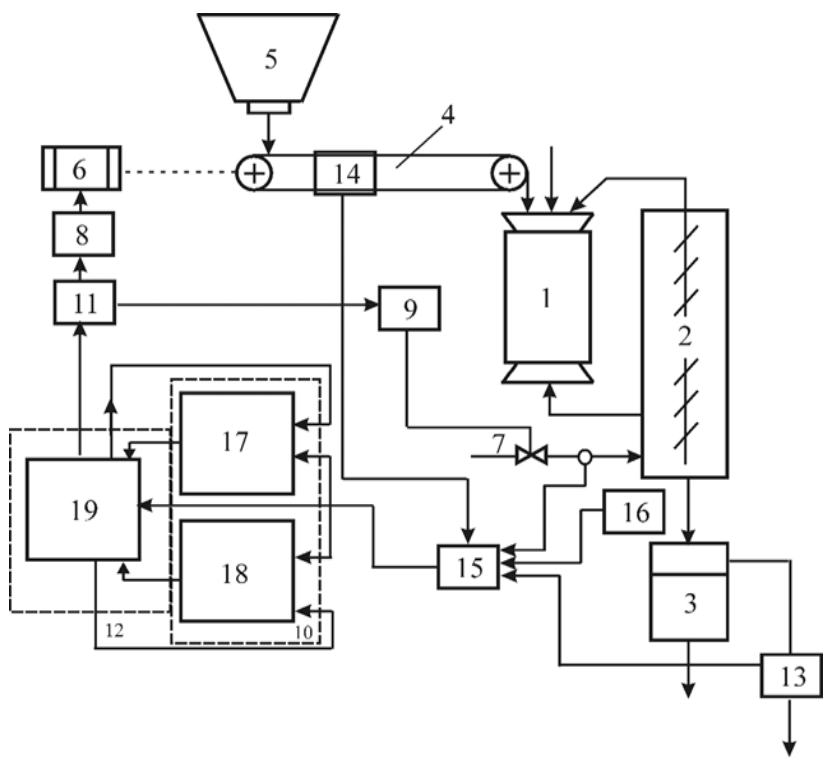

Fig. 1. The control scheme of the first stage of iron ore beneficiation

In the control circuit, we will use the combination of the control object and the inverse fuzzy model obtained by inversion of the fuzzy static block from the Hammerstein hybrid model. If we consider that the control object $(\mathrm{CO})$ is identical to the Hammerstein hybrid model and its static nonlinearity corresponds to a fuzzy block, then the set of $\mathrm{CO}$ and the inverse fuzzy block can be considered as a linear dynamic block of the Hammerstein hybrid model. In this control scheme, the calculation results of $\hat{y}$ corresponding to the linear block of the Hammerstein model is equal to the real initial variables y of $\mathrm{CO}$. Based on the differences in 'model - real object', the control system is adapted. The proposed approach allows building control based on a standard linear generalized predictive controller (GPC). As follows from the studies published in Clarke et al. (1989), Fruzetti (1997), Garcia and Morari (1982), in the case of fuzzy modeling of systems such as one input - one output or multiple inputs - one output, the predictive GPC controller allows the efficient control. The proposed control circuit allows avoiding non-linear programming in the control algorithm or the use of linearization methods.

\section{PROBLEM DEFINITION}

When implementing a control algorithm, a sequence of controls is calculated $\{\Delta u(k+j)\}, j=1, \ldots, H_{c}$, which minimizes the cost function (Stoica, 1981):

$J\left(H_{p 1}, H_{p 2}, H_{p c}, \lambda\right)=\sum_{j=H_{p 1}}^{H_{p 2}}(w(k+j)-\hat{y}(k+$ $j))^{2}+\lambda \sum_{j=1}^{H_{c}} \Delta u^{2}(k+j-1)$

where, $\hat{y}(k+j)$ are the process output parameters according to model; $w(k+j)$ are the modified state parameters, which are known in advance; $H_{p 1}$ is the minimum estimated horizon; $H_{p 2}$ maximum estimated horizon (forecast horizon); $H_{c}$ is the control horizon; $\lambda=\lambda_{0} K(u(k))^{2}$ is the limiting factor (Clarke and Mohtadi, 1989); $K(u(k))$ is the static transfer coefficient of a fuzzy block of the Hammerstein model:

$K(u(k))=\frac{\partial f(u(k))}{\partial u(k)}$.
Then, considering (1), $\Delta v(k) \approx K(u(k)) \Delta u(k)$ and (2) can be represented as:

$J\left(H_{p 1}, H_{p 2}, H_{p c}, \lambda\right) \approx \sum_{j=H_{p 1}}^{H_{p 2}}(w(k+j)-\hat{y}(k+$

$j))^{2}+\lambda_{0} \sum_{j=1}^{H_{c}} \Delta v^{2}(k+j-1)$,

Thus, the linear dynamic model gives the output parameters $\hat{y}(k+j)=\sum_{j=1}^{j} g_{i} \Delta v(k+i-1)+p_{j}$, based on which the GPC calculates the sequence of control actions $\{\Delta u(k+j)\}$; $j=1, \ldots, H_{c}$. At the same time, the response of the linear model on $k+j$ step $p_{j}$ is determined based on the control signal in the previous steps and the value $g_{i}$ :

$p_{j}=\sum_{m=1}^{j} \sum_{i=m+1}^{N_{g}} g_{i} \Delta v(k+m-i), j=1, \ldots, N_{g}$,

$g_{j}\left\{\begin{array}{c}0, \forall j \leq n_{d} \\ -\sum_{i=1}^{j} a_{i} g_{j-i}+\sum_{i=1}^{j} b_{i}, f>n_{d}\end{array}\right.$,

where, $N_{g}$ is the model horizon (Botto, 1999).

All predictable outputs can be formed into a vector $\hat{y}=$ $\left[\hat{y}\left(k+H_{p 1}\right), \ldots, \hat{y}\left(k+H_{p 2}\right)\right]$, and write the basic equation of the GPC algorithm:

$\hat{y}=G \Delta v+p$,

where,

$p=\left[p_{H_{p 1}}, p_{H_{p 1+1}}, \ldots, p_{H_{p 2}}\right]^{T}, G-$ is the matrix $\left(H_{p 2}-H_{p 1}+\right.$

1) $\times H_{l}$ with zero components for $j-i>H_{p 1}$ :

$G=\left[\begin{array}{cccc}g_{H_{p 1}} & g_{H_{p 1-1}} & \ldots & 0 \\ g_{H_{p 1+1}} & g_{H_{p 1}} & g_{H_{p 1-1}} & \vdots \\ \vdots & & \ddots & \vdots \\ g_{H_{p 2}} & g_{H_{p 2-1}} & \cdots & g_{H_{p 2}-H_{C}+1}\end{array}\right]$.

If we do not consider the restrictions, then the optimal control can be calculated analytically from (4):

$\Delta \bar{v}\left(G^{T} G+\lambda_{0} I\right)^{-1} G(w-p)$.

If constraints are taken into account, we have to use quadratic programming to solve the optimization problem. To avoid this, a two-stage approach to the constraints of the optimization problem with restrictions is proposed. The optimal control problem is solved without considering the constraints, and for the obtained predictive controls, the fulfilment of the constraints is checked. If the result is positive, then the resulting controls are applied to the system. Otherwise, it is necessary to linearize the constraints and then calculate the optimal control by one of the known methods. Such a solution procedure is more computationally rational compared to the standard ones.

Let's consider the solution of the optimization problem (3) with prediction $\Delta \bar{v}$ in the presence of restrictions in the form of equality $M \Delta \bar{v}=k$ and restrictions in the form of inequality $L \Delta \bar{v} \leq c$.

In this case, a limited solution $\Delta v^{c}$ can be found in the form:

$\Delta v^{c}=\Delta \bar{v}-\left(G^{T} G+\lambda_{0} I\right)^{-1} M^{T} \mu-\left(G^{T} G+\lambda_{0} I\right)^{-1} \cdot L^{T} \eta$,

where, $\mu$ and $\eta$ are the Lagrange multipliers vectors, corresponding to restrictions in the form of equalities and inequalities. 
We can find these vectors by solving a quadratic programming problem (Stoica, 1981):

$\min _{\mu, \eta}\left\{\left[\begin{array}{l}\mu \\ \eta\end{array}\right]^{T}+H\left[\begin{array}{l}\mu \\ \eta\end{array}\right]+g^{T}\left[\begin{array}{l}\mu \\ \eta\end{array}\right]\right\}$

Where, $H=\left[\begin{array}{cc}M\left(G^{T} G+\lambda_{0} I\right)^{-1} M^{T} & M\left(G^{T} G+\lambda_{0} I\right)^{-1} L^{T} \\ L\left(G^{T} G+\lambda_{0} I\right)^{-1} M^{T} & L\left(\left(G^{T} G+\lambda_{0} I\right)^{-1} L^{T}\right)\end{array}\right]$; $g=\left[\begin{array}{c}k-M \Delta \bar{v}^{f} \\ c-L \Delta \bar{v}^{f}\end{array}\right]$

At the same time, $\eta$ is limited by positive evaluations. When solving practical problems, it is necessary from the limitations given for $u(k)$ and $\Delta u(k)$, go to $v(k)$ and $\Delta v(k)$, using dependencies $v(k)=f(u(k))$ and $\Delta v(k) \approx K(u(k)) \Delta u(k)$. For example, from restrictions in the form:

$\left(\begin{array}{c}I_{\Delta u} \\ -I_{\Delta u} \\ I_{H_{c}} \\ -I_{H_{c}}\end{array}\right) \Delta u \leq\left(\begin{array}{c}u_{\max }-I_{u} u(k-1) \\ -u_{\min }+I_{u} u(k-1) \\ \Delta u_{\max } \\ -\Delta u_{\min }\end{array}\right)$,

we can go to restrictions $L \Delta v \leq c$ in the form:

$\left(\begin{array}{c}I_{\Delta u} \\ -I_{\Delta u} \\ I_{H_{c}} \\ -I_{H_{c}}\end{array}\right) \Delta v \leq\left(\begin{array}{c}v_{\max }-I_{u} v(k-1) \\ -v_{\min }+I_{u} v(k-1) \\ \Delta \bar{v}_{\text {max }} \\ -\Delta \bar{v}_{\min }\end{array}\right)$,

where, $\quad v_{\min }=f\left(u_{\min }\right), \quad v_{\max }=f\left(u_{\max }\right), \quad \Delta \bar{v}_{\min }=$ $\Delta v_{\text {min }} K, \Delta \bar{v}_{\text {max }}=\Delta v_{\text {max }} K, \quad K=[K(u(k)), \ldots, K(u(k+$ $\left.\left.\left.H_{c}\right)\right)\right]$.

In order to calculate $\Delta v_{\min }$ and $\Delta v_{\max }$, it is necessary to know the vector $K$, caused by the sequence of predicted controls, which are unknown at the time of calculations in step $k$. To determine this sequence, we can use algorithms, which are similar to the limited control method for linearized feedback.

Let's consider two algorithms for determining the control sequence (operating trajectory) for the fuzzy Hammerstein model with inversion of static nonlinearity. The first method allows choosing the optimal operating trajectory, considering the constraints imposed on it by inequality (8).

\section{Algorithm 1.}

1. Determine unlimited optimal control $\Delta \bar{v}$, by solving (5).

2. Transform $\Delta \bar{v}$ and $\Delta u$ through non-linear mapping inversion $f^{-1}$.

3. Check the resulting control sequence for compliance with the constraints (7). If the constraints are satisfied, the resulting control is applied to the system in accordance with the methodology of the MPC (principle of prediction horizon, which moves away). If the restrictions are not met, then go to step 4.

4. Linear constraints are calculated in the form of inequalities (8), approximating the vector $\mathrm{K}$ with respect to the initially $\mathrm{ob}$ tained sequence of controls.

5. Optimal forecast $\Delta \bar{v}^{c}$, calculated according to (6), using precomputed constraints (Step 4).

Go to step 2.

Studies of similar algorithms show that the algorithm should converge to a suboptimal solution (Morkun et al., 2014b). If the resulting sequence of controls is not feasible, then it is recommended to strengthen the restrictions so that the solution is guaranteed to be feasible. To do this, take $\Delta v_{\min }=\Delta v_{\min } K_{\max }$ and
$\Delta v_{\max }=\Delta v_{\max } K_{\min }$, wherein $K_{\min }$ and $K_{\max }$ are calculated as follows:

$K_{\min , i}=\min _{u} K(u)$,

$\forall u \in\left[u(k-1)-i \Delta u_{\min }, u(k-1)+i \Delta u_{\max }\right]$,

$K_{\max , i}=\max _{u} K(u)$,

$\forall u \in\left[u(k-1)-i \Delta u_{\min }, u(k-1)+i \Delta u_{\max }\right]$.

Now for optimal prediction $\Delta \bar{v}^{c}$, found from (6), the restriction $L \Delta \bar{v} \leq c^{*}$ is applied in accordance with (7) and (8), which ensures the known feasibility of the solution. The proposed algorithm may increase the constraints imposed on the control too much. Therefore, as an alternative variant, it is possible to propose a different algorithm, which adapts NOT for control, but control constraints (in the form of linear inequalities).

\section{Algorithm 2.}

1. Calculate the constraints $c^{*}$ similar to (7) considering (9).

2. Determine the vector $\hat{c}$ as the initial approximation of the constraints, calculated in accordance with Step 4 of Algorithm 1 for the last iteration.

3. Set new restrictions $L \Delta \bar{v} \leq c$, where $c$ is a linear combination $c^{*}$ and $\hat{c}$, that is,

$c=\eta c^{*}+(1-\eta) \hat{c}(\eta$ - a certain step size $)$.

4. Calculate the optimal prediction $\Delta \bar{v}^{c}$ according to (6), considering (8), and transform $\Delta \bar{v}^{c}$ to the real sequence of controls $\Delta u$ (by inverse nonlinear mapping).

5. If restrictions (7) are not violated, apply to the system the received controls in accordance with the MPC procedure. Otherwise, go to Step 3. The scheme of this algorithm is shown in Fig. 2. to (5):

The determination of unlimited optimal control $\Delta v^{f}$ according

$\Delta v^{f}=\left(G^{T} G+\lambda_{0} I\right)^{-1} G(w-p)$,

where $\Delta v=\left[\Delta v(k), \ldots, \Delta v\left(k+H_{c}\right)\right]^{T}$,

$p=\left[p_{H p 1}, p_{H p 1+1}, \ldots, p_{H p 2}\right]^{T}$,

$p_{j}=\sum_{m=1}^{j} \sum_{i=m+1}^{N_{g}} g_{i} \Delta v(k+m-i), j=1, \ldots, N_{g}$, is per-

formed in block 6 .

Then the resulting control sequence $\Delta v^{f}$ let's transform into a sequence of real controls $\Delta u$ by the non-linear mapping inversion $f^{-1}$ (block 8).

Block 9 is used to verify the received control sequence for compliance with the restrictions (7). If the restrictions are satisfied, then the resulting control is applied to the system in accordance with the predictive control methodology based on the MPC model. If the restrictions are not met, then the parameters of the fuzzy block in the hybrid model of Hammerstein are specified in accordance with (8) according to the initial control sequence (block 11).

Block 12 according to the updated data calculates a new prediction $\Delta v^{f c}$ according to (6) $\Delta v^{c}=\Delta v^{f}=\left(G^{T} G+\right.$ $\left.\lambda_{0} I\right)^{-1} M^{T} \mu-\left(G^{T} G+\lambda_{0} I\right)^{-1} L^{T} \eta$, by which the specified sequence of controls is determined. If $\eta=1$, then we get a case of severe restrictions corresponding to the worst-case analysis, which gives a guaranteed feasible solution for control and convergence of the procedure. 


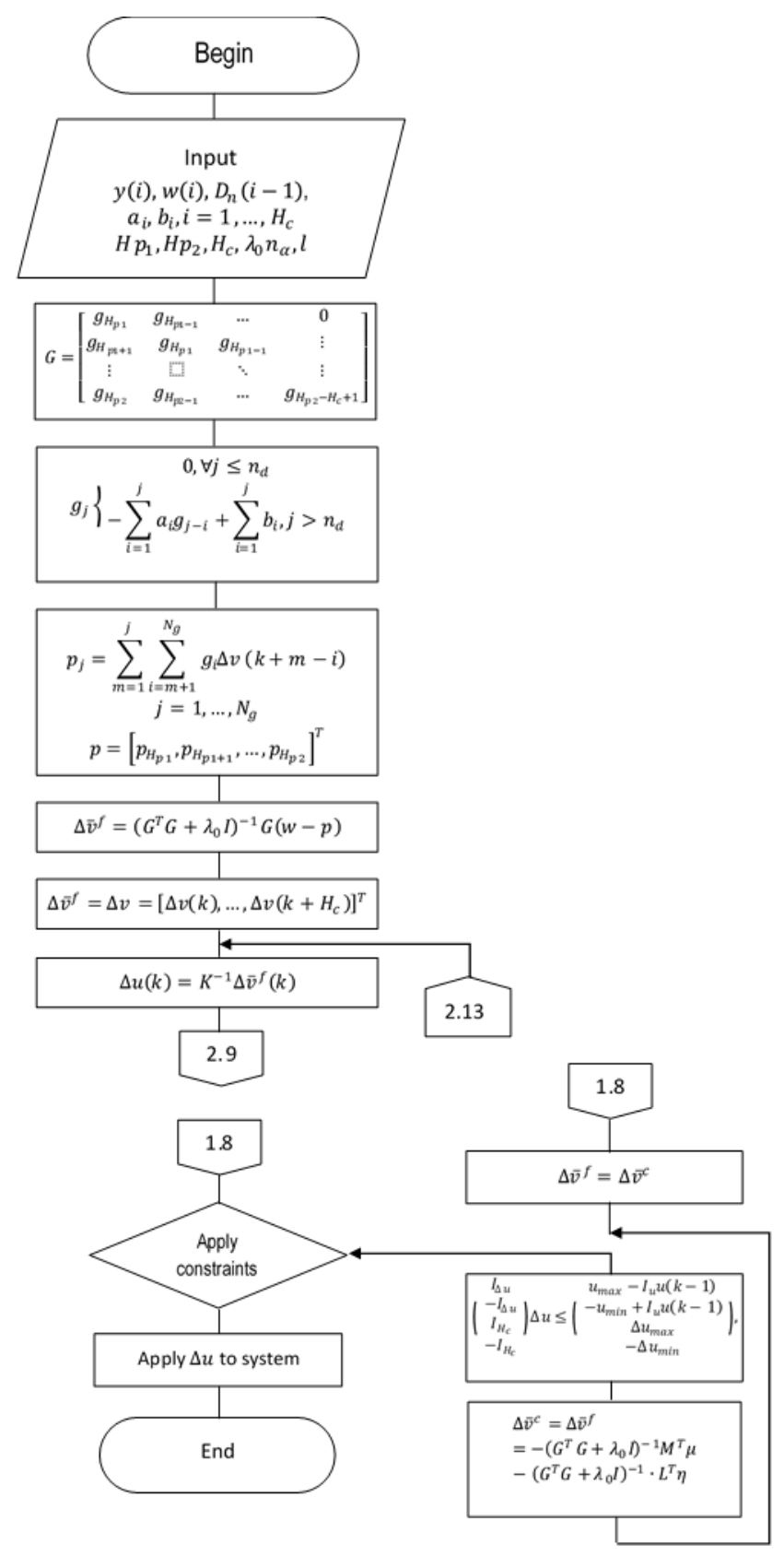

Fig. 2. Scheme of the control algorithm

\section{RESULTS AND ANALYSIS}

When implementing the control according to the scheme shown in Fig. 1 , the value of the mill productivity by ore $u_{1}=Q_{1}$ and water flow rate in the classifier $u_{2}=Q_{2}$ were used as control actions. As output indicators - performance on the intermediate product $y_{1}=Q_{p r}$, iron content in the solid phase of the intermediate product $y_{2}=\beta_{p r}$ and the loss of a useful component in the tails $y_{3}=\beta_{t}$ were used. In the course of control, the water flow rate into the mill was calculated while observing the ratio of ore to water within the limits of given parameters.

The control actions were formed based on measuring the iron content of the magnetic and solid phases in the pulp (sensors 13, 16), the concentration of particles of control size grades in the pulp at the discharge of classifier 2 (sensor 16), processed by the information support subsystem 15, the structure and operation principles of which are described in Morkun et al. (2015b).
For approximation, a discrete model with a fuzzy nonlinear block was used. For each input variable, three terms were applied, according to the Hammerstein hybrid model. Three terms with kernels of $16 \% ; 24 \% ; 32 \%$ for the pulp density of the classifier discharge and two terms with kernels of 220; 230; 240 for mill productivity by ore.

According to the measured input and output variables, the subsystem 10 identifies the control object, builds the Hammerstein hybrid model. The linear dynamic block is approximated by a model:

$\hat{y}(k+1)=$

$\sum_{i=1}^{n_{y}} A_{i} \hat{y}(k-i+1)+$

$\sum_{i=1}^{n_{u}} \sum_{j=1}^{N_{1}} \sum_{l=1}^{N_{2}} \sum_{p=1}^{N_{3}} B_{i} d_{j l p} \beta_{j l p}\left(u\left(k-i-n_{d}+1\right)\right)$,

where, matrices $A_{i}$ and $B_{i}$ for the considered case, have dimension $3 \times 3, N$ is the number of fuzzy sets for the corresponding input $\left(N_{1}=3, N_{2}=3, N_{3}=3\right), \hat{y}(k+i)$ is the vector of output indicators (three components of the vector $-y_{1}=Q_{p r}$, $y_{2}=\beta_{p r}, y_{3}=\beta_{t}$ )

By measurements of input and output indicators step by step, according to algorithms, matrices $A_{i}, B_{i}$, coefficients $d_{j}$ the values $\beta_{j}$ are determined. The resulting model is specified at each step (see block diagram in Fig. 2). The optimizer 12 operates on the principle of predictive control with an inverse fuzzy model. In order to obtain optimal control effects in accordance with the obtained model, a minimum target function is found:

$J\left(H_{p 1}, H_{p 2}, H_{p c}, \lambda\right) \approx \sum_{i=H_{p 1}}^{H_{p 2}}(y(k+i)-\hat{y}(k+$ $i))^{2}+\lambda_{0} \sum_{i=1}^{H_{c}} \Delta v^{2}(k+i-1)$,

where, $y(k+i)$ are the set values of output variables; $\hat{y}(k+i)$ are the output variable values predicted by the model; $\Delta v(k+$ $i-1)$ are the control actions determined from the condition of the minimum of the target function.

This considers the restrictions imposed on $\Delta v$, according to (7). Based on $\hat{y}(k+i)$, the predictive controller calculates the sequence of control impacts. The target function is quadratic, so the problem was considered as quadratic programming and solved using the simplex method. The resulting controls were applied to the object in order to obtain at the output of $y_{1}$ a consistently high value of the intermediate product output and at the output of $y_{2}$ a stable specified value of the iron content in the intermediate product. Using the simplex method in accordance with the requirements $Q_{\text {int }} \rightarrow \max , \beta_{1} \leq \beta_{p r} \leq \beta_{2}$, we previously found the maximum performance $y_{1}=Q_{p r}$ on the intermediate product with imposed restrictions on quality $52 \% \leq$ $y_{2} \leq 55 \%$.

The estimation of the quantity of the output product of a closed grinding cycle (discharge of a hydro cyclone) $Q(t)$ with a given particle size characteristic in the presence of disturbances in the inputs $w$ and measurements $v$ is shown in Fig 3 . The results of $Q(t)$ prediction, obtained based on the developed hybrid fuzzy model are shown in Fig. 4. The standard deviation between accurate and formed estimates of the values of $Q(t)$ is 0.57 .

The results of the research were implemented at the enterprises of the Kryvyi Rih iron ore basin, which are part of the Ukrrudprom Association, which allows adequate structural and parametric identification of control objects (determination coefficient $R^{2} \geq 0.96$, identification error is in the range 0.005-0.067). This made it possible to maximize the productivity of technological units, increase the content of the useful component in the concen- 
trate by $0.15 \%$ and reduce energy consumption by $1.85 \%$. Tests have shown that the use of control algorithms based on hybrid models reduces the variance of parameters (by more than $27 \%$ ) and the duration of transients (by more than $22 \%$ ).

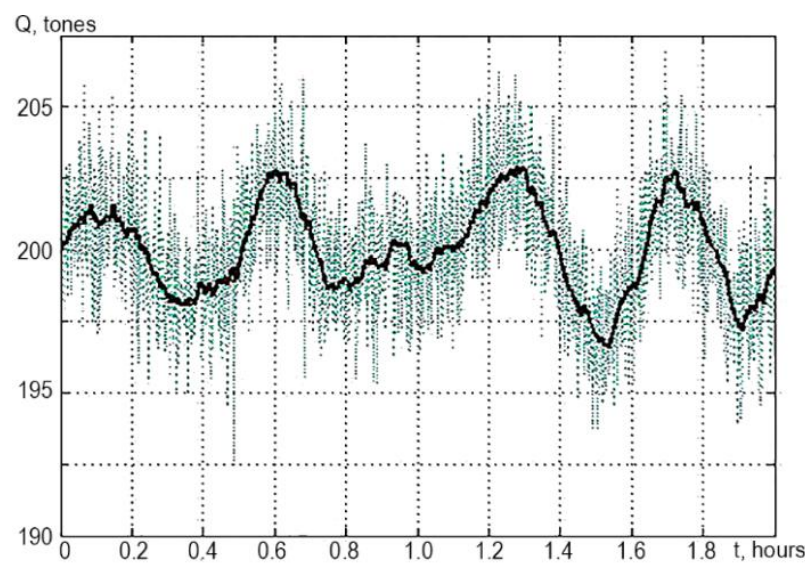

Fig. 3. Estimation of the quantity of the output product of a closed grinding cycle

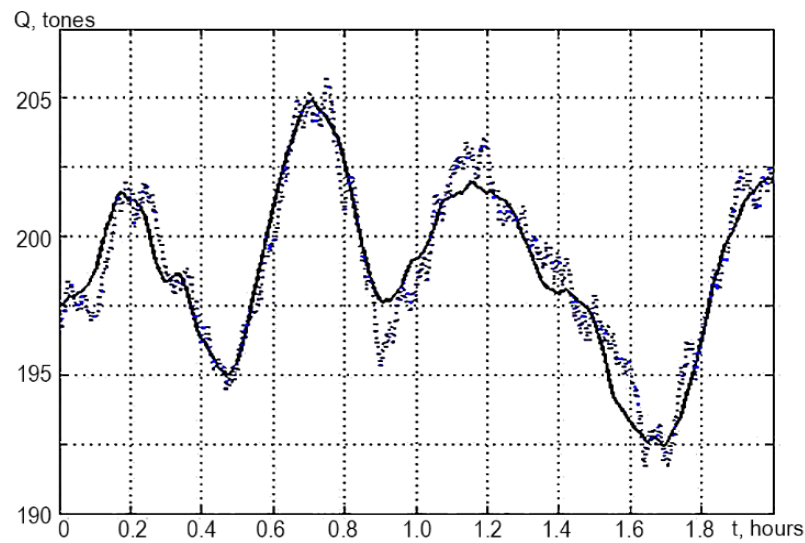

Fig. 4. The results of $\boldsymbol{Q}(\boldsymbol{t})$ prediction, obtained based on a hybrid fuzzy model

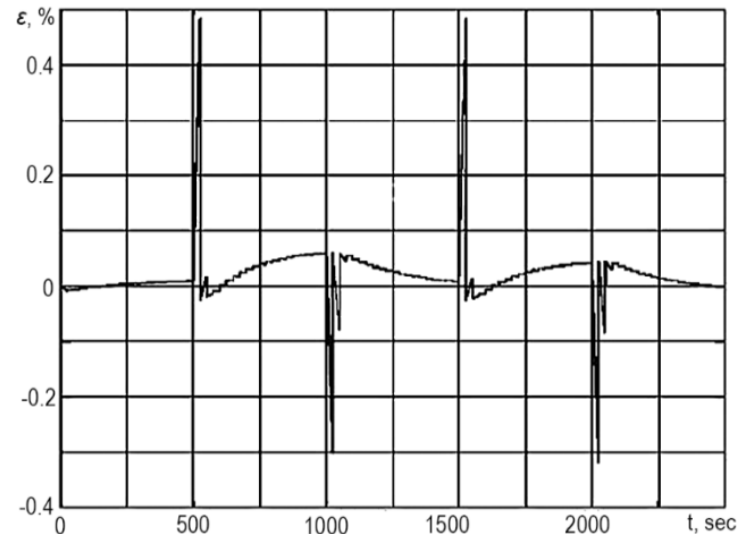

Fig. 5. The change in control error when changing the value of the setting of the ore grinding degree in the hydrocyclone discharge

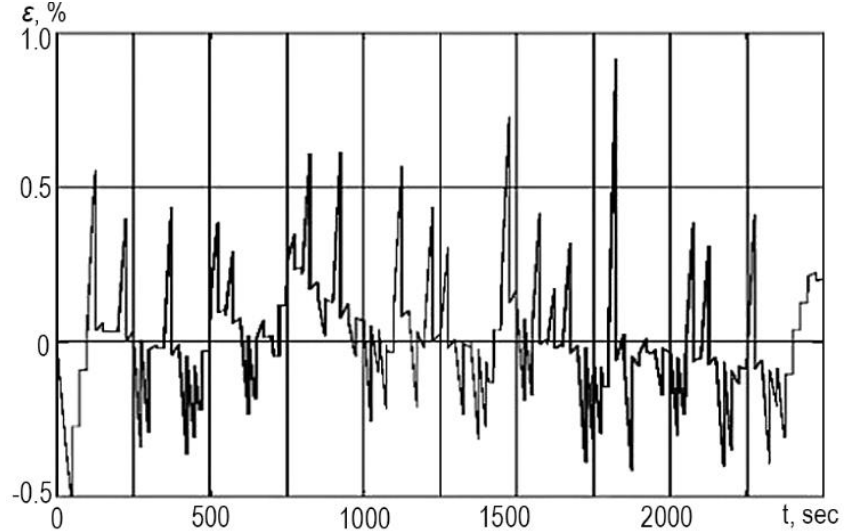

Fig. 6. Change of regulation error when changing the particle size distribution of the pulp solid phase in the mill discharge

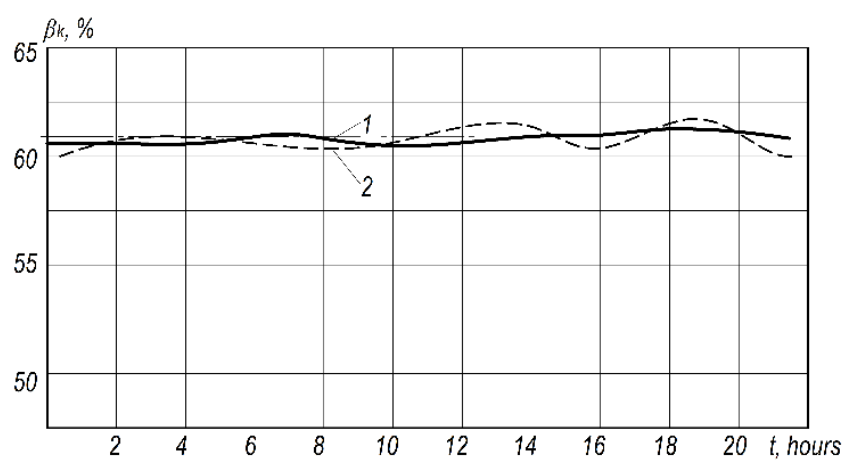

Fig. 7. Graphs of changes in the magnetic iron content in the intermediate product of the steady-state iron ore processing line: 1 - the control system based on the Hammerstein hybrid model; 2 - the control system with reconfigurable PID controllers

The effectiveness of the considered control system was evaluated concerning the hierarchical structure based on local automatic control systems with reconfigurable PID controllers and the calculation of optimal tasks for these systems. Figure 7 shows graphs of changes in the magnetic iron content in the intermediate product of the steady-state iron ore processing line.

Thus, the proposed algorithm based on the Hammerstein hybrid model ensures convergence of the procedure and ensures efficient control of the objects under consideration

\section{CONCLUSIONS}

1. Non-linear, dynamic, non-stationary properties characterize objects of the iron ore beneficiation line; therefore, it is advisable to use models of the Hammerstein class for their approximation. The hybrid model of Hammerstein, in which the nonlinear block is represented by fuzzy Takagi-Sugeno models of zero and first order, built based on linguistic rules about the control object, makes it possible to visually and conveniently approximate the control object during magnetic beneficiation of iron ores. Therefore, it is advisable to use such models as predicting when controlling the beneficiation processes according to the principles of MPC in the context of incomplete and fuzzy information about the state of the control object.

2. The developed algorithms allow identification by automatically extracting information from a fuzzy knowledge base about the ore beneficiation process, which allows them to be used for 
real-time identification. The proposed identification mechanisms based on Hammerstein's hybrid models allow us to avoid non-linear optimization with non-linear res, which greatly simplifies the process of model coefficients calculating. The restrictions imposed on the model parameters guarantee the convergence of the procedure.

3. The application of the model fuzzy block inversion in the synthesis of predictive control based on the Hammerstein hybrid model allows determining the prediction based on the linear model, which greatly simplifies the calculation of optimal control actions.

4. The proposed Hammerstein hybrid model makes it possible to consider the information of various mathematical classes for the implementation of adequate structural and parametric identification of the control objects of the beneficiation production (determination coefficient $R^{2} \geq 0.96$. Depending on the dynamic characteristics of the object, the relative error of identification varies from 0.005 to 0.067 ; therefore, models allow the approximation of control objects with various dynamic properties, which are used at processing plants.

\section{REFERENCES}

1. Abba S.I., Nourani V., Elkiran G. (2019), Multi-parametric modeling of water treatment plant using Al-based non-linear ensemble, Aqua, 68 (7), 547-561.

2. Abonyi J., Nagy L., Szeifert F. (2000), Hybrid fuzzy convolution modelling and identification of chemical process systems, International Journal of Systems Science, 31, 457-466.

3. Babuska R. (1998), Fuzzy Modeling for Control, Kluwer Academic Publishers, Boston.

4. Babuska R., Roubos J.A., Verbruggen H.B. (1998), Identification of MIMO systems by input-output TS fuzzy models, Proceedings FUZZ-IEEE'98, Anchorage, Alaska, 57-69.

5. Botto M. Ayala, van den Boom T.J.J., Krijgsman A., S'a da J. (1999), Costa Constrained nonlinear predictive control based on input-output linearization using a neural network, International Journal of Control, 72(17), 1538-1554.

6. Chen H., Ding F. (2015), Hierarchical least-squares identification for Hammerstein nonlinear controlled autoregressive systems, Circuits, Systems and Signal Processing, 34(1), 61-75.

7. Chen J., Wang X. (2015), Identification of Hammerstein systems with continuous nonlinearity, Information Processing Letters, 115(11), 822-827.

8. Clarke D. W., Tuffs P.S., Mothadi C. (1989), Generalized predictive control - part I. the basic algorithm, Automatica, 23, 137-148.

9. Clarke D.W., Mohtadi C. (1989), Properties of generalized predictive control, Automatica, 25(6), 859-875.

10. Falck T., Dreesen P., Brabanter K., Pelckmans K., De Moor B., Suykens J.A.K. (2012), Least-Squares Support Vector Machines for the identification of Wiener-Hammerstein systems, Control Engineering Practice, 20(11), 1165-1174.

11. Fruzetti K.P., Palazoglu A., McDonald K.A. (1997), Nonlinear model predictive control using Hammerstein models, Journal of Process Control, 7(1), 31-41.

12. Garcia C.E., Morari M. (1982), Internal model control: 1.A unifying review and some new results, Ind. Eng. Chem. Process Res. Dev, $21,308-323$

13. Ikhouane F., Giri F. (2014), A unified approach for the parametric identification of SISO/MIMO Wiener and Hammerstein systems, Journal of the Franklin Institute, 351(3), 1717-1727

14. Ivanov A.I. (1991), Ortogonalnaya identifikatsiya nelineynykh dinamicheskikh sistem s konechnoy i beskonechnoy pamyatyu pri odnom i neskol'kikh vkhodakh [Orthogonal identification of nonlinear dynamical systems with finite and infinite memory at one or several inputs], NIKIRET, Penza (In Russian),
15. Ivanov A.I. (1995), Bystryye algoritmy sinteza nelineynykh dinamicheskikh modeley po eksperimental'nym dannym [Fast algorithms for the synthesis of nonlinear dynamic models from experimental data], NPF "Kristall", Penza (In Russian),

16. Junhao Shi, Sun H.H. (1990), Nonlinear system identification for cascaded block model: an application to electrode polarisation impedance, IEEE Trans. Biomed. Eng, 6, 574-587.

17. Kazuo T., Wang H.O. (2001), Fuzzy Control Systems Design And Analysis, John Wiley\&Sons.

18. Le F., Markovsky I., Freeman C.T., Rogers E. (2012), Recursive identification of Hammerstein systems with application to electrically stimulated muscle, Control Engineering Practice, 20(4), 386-396

19. Leontaritis I.J. Billings S.A. (1987), Experimental design and identifiably for nonlinear systems, International Journal of Systems Science, 18, 189-202.

20. Li Yu., Shchetsen M. (1968), Opredeleniye yader Vinera-Khopfa metodom vzaimnoy korrelyatsii. Tekhnicheskaya kibernetika za rubezhom [Determination of Wiener-Hopf kernels by crosscorrelation. Technical cybernetics abroad], Mashinostroyeniye, Moskow (In Russian),

21. Ma J., Ding F., Xiong W., Yang E. (2016), Combined state and parameter estimation for Hammerstein systems with time-delay using the Kalman filtering, International Journal of Adaptive Control and Signal Processing, 00:1-17. DOI: 10.1002/acs

22. Mete S., Ozer S., Zorlu H. (2016), System identification using Hammerstein model optimized with differential evolution algorithm, AEU International Journal of Electronics and Communications, 70(12), 1667-1675.

23. Morkun V., Morkun N., Pikilnyak A. (2014a), Ultrasonic phased array parameters determination for the gas bubble size distribution control formation in the iron ore flotation, Metallurgical and Mining Industry, 6(3), 28-31.

24. Morkun V., Morkun N., Pikilnyak A. (2014b), Ultrasonic facilities for the ground materials characteristics control, Metallurgical and Mining Industry, 6(2), 31-35

25. Morkun V., Morkun N., Pikilnyak A. (2014c), The adaptive control for intensity of ultrasonic influence on iron ore pulp, Metallurgical and Mining Industry, 6, 8-11.

26. Morkun V., Morkun N., Pikilnyak A. (2015c), Adaptive control system of ore beneficiation process based on Kaczmarz projection algorithm, Metallurgical and Mining Industry, 2, 35-38.

27. Morkun V., Morkun N., Tron V. (2015a), Formalization and frequency analysis of robust control of ore beneficiation technological processes under parametric uncertainty, Metallurgical and Mining Industry, 5, 7-11.

28. Morkun V., Morkun N., Tron V. (2015b), Model synthesis of nonlinear nonstationary dynamical systems in concentrating production using Volterra kernel transformation, Metallurgical and Mining Industry, $10,6-9$.

29. Morkun V., Morkun N., Tron V., Hryshchenko S. (2018), Synthesis of robust controllers for the control systems of technological units as iron ore processing plants, Eastern European Journal of Enterprise Technologies, 1(2-91), 37-47.

30. Morkun V., Tcvirkun S. (2014), Investigation of methods of fuzzy clustering for determining ore types, Metallurgical and Mining Industry, 5, 11-14.

31. Narendra K.S., Gallman P.G. (1966), An iterative method for the identification of nonlinear systems using the Hammerstein model, IEEETrans. Automatic Control, 12, 546.

32. Ozer S., Zorlu H., Mete S. (2016), System identification application using Hammerstein model, Sadhana, 41(6), 597-605.

33. Piroddi L., Farina M., Lovera M. (2012), Black box model identification of nonlinear input-output models: A Wiener-Hammerstein benchmark, Control Engineering Practice, 20(11), 1109-1118.

34. Postlethwaite B.E. (1996), Building a model-based fuzzy controller, Fuzzy Sets and Systems, 79, 3-13. 
36. Rébillat M., Hennequin R., Corteel E., Katz B. (2010), Identification of cascade of Hammerstein models for the description of nonlinearities in vibrating devices, Journal of Sound and Vibration, 330(5), 1018-1038.

37. Rossiter J.A. (2003), Model-Based Predictive Control: a Practical Approach, CRC Press.

38. Sanches J.M.M., Rodellar J. (1996), Adaptive predictive control: from the concepts to plant optimization, Prentice Hall International (UK) Limited.

39. Sjoberg J., Zhang Q., Ljung L., Benveniste A., Deylon B., Glorennec P-Y., Hjalmarsson H., Juditsky A. (1995), Nonlinear blackbox modeling in system identification: a unified overview, Automatica, 31, 1691-1724.

40. Stoica P. (1981), On the convergence of an iterative algorithm used for Hammerstein system identification, IEEETrans. Automatic Control, 26, 967-969.

41. Tobi T., Hanafusa T. (1991), A practical application of fuzzy control for an airconditioning system, International Journal of Approximate Reasoning, 5, 331-348.

42. Verhaegen M., Westwick D. (1996), Identifying MIMO Hammerstein systems in the context of subspace model identification, International Journal of Control, 63, 331-349.

43. Wills A., Ninness B. (2012), Generalised Hammerstein-Wiener system estimation and a benchmark application, Control Engineering Practice, 20(11), 1097-1108.

44. Young A.D. (1977), State of the art and trends in computers and control equipment, 2-nd IFAC Symp."Automat. Mining, Miner. and Metal. Proc.", Pretoria, 41-46.

45. Yucai Z. (1999), Parametric Wiener model identification for control, Proceedings IFAC World Congress, Bejing, China, 3a-02-1, 34-46.

46. Zubov D.A. (2006), Passifikatsiya $i$ sintez algoritma avtomaticheskogo upravleniya odnim klassom SISO-obyektov ugleobogashcheniya $s$ ispolzovaniyem algebry Li [Passification and synthesis of an automatic control algorithm for one class of SISO objects for coal enrichment using Lie algebra], Zbagachennya korisnikh kopalin, 24(65), 80-87 (In Russian), 